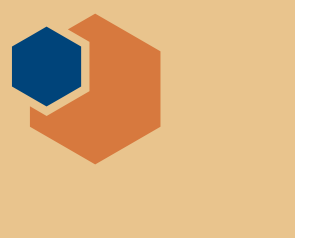

\title{
The energy of the atoms brings the stars to within reach
}

Space exploration enters a new phase, as governments worldwide expand their presence in orbit and industry launches ever-more commercial satellites and space tourists. But for science missions to reach distant worlds, spacecraft must function, undeterred, in the unforgiving environment of space. For 60 years, nuclear energy has been, and remains, a crucial solution to this challenge.

$\mathrm{O}$ October 16, 2021, NASA launched an ambitious mission to explore several asteroids in the Solar System's asteroid belt and probe their chemical composition. Shortly after launch, one of its solar panel arrays failed to latch to the body of the "Lucy" spacecraft and immediately sparked grave concerns regarding the mission's subsequent capabilities. Moreover, this technical setback underscored the biggest challenge of interstellar travel: a spacecraft cannot operate without reliable power in the harsh environment of space.

Unpiloted probes and crewed missions alike must carry all fuel that they may need from their earthbound launch pads through to their destinations, and life support systems exponentially compound this challenge. Standalone solar power is insufficient to propel heavy, fast-traveling vehicles, and capacity diminishes in the farther reaches of our solar system. Chemical rockets have finite energy densities innate to their inherent design, which has remained fundamentally unchanged since their 1940s conception. As highlighted in an October 2021 US House of Representatives "Accelerating Deep Space Travel with Space Nuclear Propulsion" Committee hearing, a human mission to Mars requires up to nine months for a one-way trip and, owing to short available launch windows, a 500-day-long stay on the surface. The energy demands for such a mission, including life support and scientific data acquisition, are immense and difficult to meet using existing solutions.

\section{Radioisotope power generation}

Space is cold and dark. Even if solar panels can capture enough sunlight to power an unmanned spacecraft's systems, battery storage of this energy will not survive freezing temperatures for long. To address this challenge, NASA has turned to radioactive materials that emit heat as they naturally decay. Thermoelectric devices convert this heat into usable electricity for the spacecraft. As a bonus, the heat can be used to keep temperature-sensitive onboard instruments warm.

The Transit IV-A Earth-orbiting satellite was launched in 1961 and carried a $2.7-\mathrm{W}$ radioisotope generator. As the first-ever spacecraft to benefit from this technology, it circled the globe 25,000 times and set a mission endurance record. In the 60 years that followed, the US Department of Energy (DOE) and NASA built upon this success and improved this technology. Over 25 missions have relied on radioisotope power systems (RPS) to date. These include many interplanetary probes, such as Pioneer,

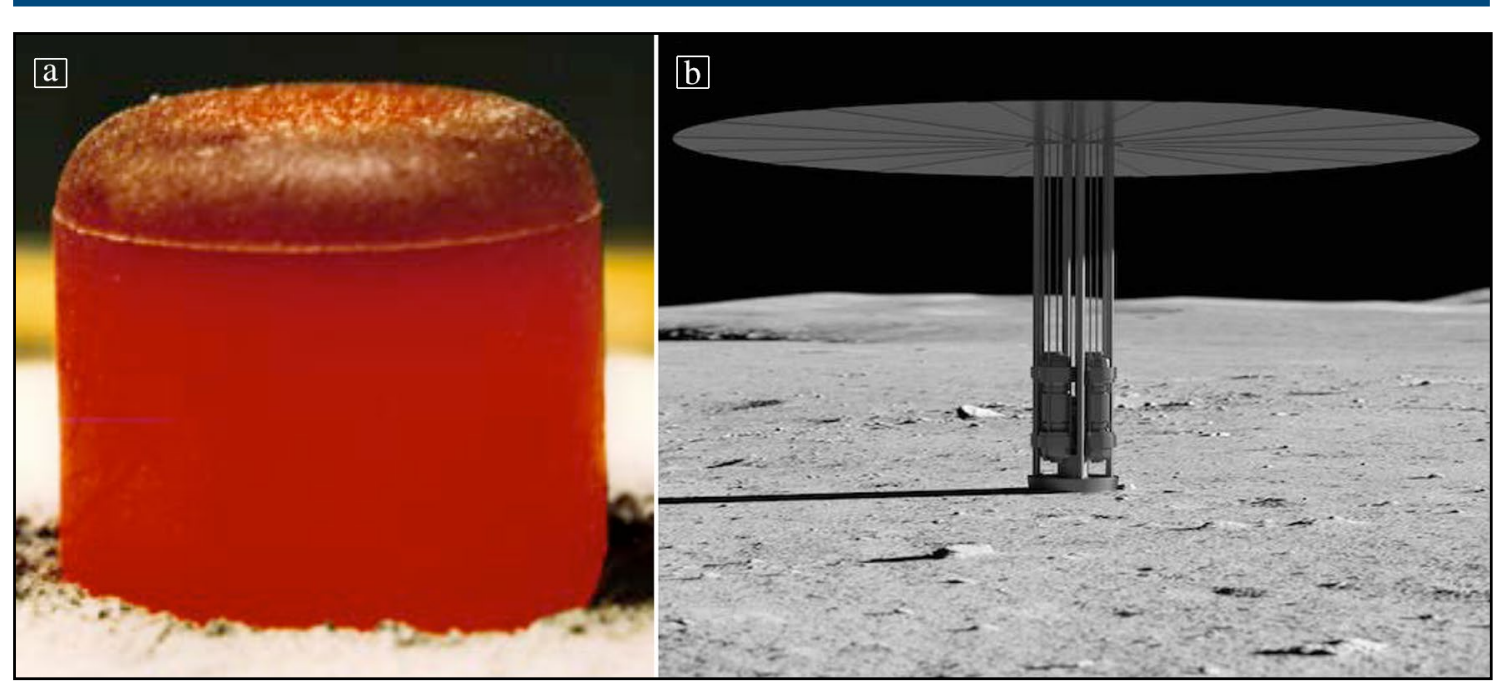

(a) Plutonium-238 ingot produced for a radioisotope power system. Credit: U.S. Department of Energy. (b) An artist's schematic of a nuclear electric propulsion fission reactor on the lunar surface. Credit: NASA. 
Cassini, and Galileo. All six Apollo Moon landings used RPS-powered surface experiment packages. Starting with the Viking missions, Mars surface landers and rovers have universally relied on radioisotope power to collect and transmit data as they explore the red planet. Most recently, the 2020-launched Perseverance Mars Rover included the multi-mission radioisotope thermoelectric generator (MMRTG), which generates $110 \mathrm{~W}$ of power (beginning-of-mission level) and is expected to last for 17 years. Dragonfly, the next mission to use a MMRTG, is slated to land on and fly around Titan and is planned for launch in 2027.

In an interview with MRS Bulletin, June Zakrajsek, manager for NASA's Radioisotope Power Systems Program office at NASA Glenn Research Center in Cleveland, highlighted the significance of using RPS and the benefits for space exploration. "RPS are reliable and efficient," said Zakrajsek. "Over the past 60 years, they've allowed us to explore extreme environments from the Sun to Pluto and beyond."

While NASA plans and executes space missions that use RPS, DOE delivers the fueled systems to NASA. The plutonium-238 isotope $(\mathrm{Pu}-238)$ has been the heat source for all NASA missions over the past decades. This non-fissile material generates a steady heat output, which has an 87.7-year half-life. According to Zakrajsek, the RPS used for missions like New Horizons decrease the power output by only $1.8-1.9 \%$ every year. This longevity has enabled missions like the Voyager probe, which has relied on a 470-W beginning-of-mission output of its RPS to operate for 44 years and leave the solar system.

Until recently, the United States has had to rely on imports from Russia as well as remnants of a 30-year-old stockpile, which had been produced at the Savannah River Nuclear Site before the end of the Cold War, for $\mathrm{Pu}-238$. However, a rejuvenated need for a domestic supply of this isotope enabled Oak Ridge National Laboratory (ORNL) to resume production. In 2015, following NASA's two-year USD \$15 million investment through the DOE Office of Nuclear Energy, the laboratory produced a 50-g quantity of the material. A 2017 presentation by DOE's Deputy Assistant Secretary for Nuclear Energy outlined the supply chain to deliver $\mathrm{Pu}-238$ for NASA's missions.

$\mathrm{Pu}-238$ production starts at ORNL with the fabrication of neptunium-237 targets using neptunium oxide supplied by Idaho National Laboratory (INL). The targets are irradiated in ORNL's High Flux Isotope Reactor and INL's Advanced Test Reactor to produce the desired $\mathrm{Pu}-238$ isotope. ORNL processes the irradiated targets, while Los Alamos National Laboratory subsequently manufactures fueled clads using this material, and INL completes the production of general-purpose heat source modules. INL also stores them, in anticipation of their allocated NASA missions, and completes final fueling, assembly, and testing of the RPS. DOE is continually scaling this process up across its national laboratories and aims to deliver $1.5 \mathrm{~kg}$ annually by 2026 .

The manufactured $\mathrm{Pu}-238$ material must be at least $82.5 \%$ pure; removal of the uranium-234 decay byproduct is especially important. ORNL's Radiochemical and Engineering Center completes this refinement and chemical processing. Moreover, batch-to-batch powder morphology and crystallinity control are essential for manufacture of $\mathrm{Pu}-238$ into stable ceramic pellets that deliver optimal performance in NASA's RPS. DOE's goals to successfully scale up its production are contingent on sustained scientific development, including new irradiation target designs, investments in fuel cladding manufacturing, and evaluation of new technology that builds resilience into the overall supply chain.

The NASA-DOE interagency agreement has been codified through several memoranda of understanding (MOUs). The first such agreement was signed in 1965, and a 2016 iteration updated its terms. Under the most recent MOU, NASA develops the mission requirements for spacecraft that require RPS, while DOE designs the actual energygenerating device and produces the corresponding nuclear fuel. Under the agreement, NASA funds DOE's work. A recent NASA white paper estimated USD\$75 million as the annual cost of the overall RPS program, while a 2017 US Government Accountability Office report estimated that USD $\$ 50$ million of those expenditures cover DOE's share of the work.

As space missions aim to accomplish more challenging milestones, the NASA-DOE relationship will remain crucial for the RPS technology to evolve and meet these goals. Zakrajsek outlined two of these priorities. First, development of novel thermoelectric materials may increase efficiencies and decrease power degradation. Second, next-generation RPS, which may enable more versatile energy conversion beyond thermoelectric conversion, may become more efficient and demand less fuel. DOE's capabilities, from basic research conducted in its national laboratories to radioisotope production, will remain integral to these efforts.

\section{Nuclear propulsion}

Although radioisotope technology may provide sufficient power and warmth to electrical instruments onboard small, unmanned drones, RPS modules alone cannot overcome limitations of slow interplanetary travel. Nuclear power may advance peak velocities of spacecraft well beyond the capabilities offered by conventional chemical rockets or ion thrusters. A 2021 National Academy of Sciences Report, Space Nuclear Propulsion for Human Mars Exploration, outlined two main technologies that nuclear reactors may enable onboard space vehicles. Nuclear thermal propulsion (NTP) aims to directly accelerate spacecraft with 
a generated impulse (unit of rocket thrust divided by propellant flow rate) of at least $900 \mathrm{~s}$. Conversely, nuclear electric propulsion (NEP) provides a space vehicle with at least $1 \mathrm{MW}$ of onboard electric power and higher energy-to-weight ratio than all other energy-storage solutions (such as batteries or fuel cells). A NTP reactor heats compressed hydrogen, which flows to the reactor from a cryogenic storage tank, and the superheated $\left(2500^{\circ} \mathrm{C}\right)$ gas escapes the rocket nozzle to accelerate the spacecraft.

Shortly after nuclear power generation entered mainstream use half a century ago, DOE became involved in nuclear reactor and fuel development to meet NASA's ambitious space exploration goals. From 1955 until 1973, the Atomic Energy Commission (DOE's predecessor) developed a nuclear reactor for NTP under its Project Rover, while NASA's 1961-1973 Nuclear Engine for Rocket Vehicle Applications (NERVA) Program aimed to integrate the reactor into an operational propulsion system. Despite extensive testing and development, shifts in funding priorities canceled both efforts before they could be realized. The United States undertook a single orbital test of the SNAP$10 \mathrm{~A}$ reactor, which generated $500 \mathrm{~W}$ of electrical power.

The goal of sending a crewed mission to Mars renewed interest and funding for both nuclear power concepts. NASA manages both NTP and NEP development efforts through its Space Technology Mission Directorate, which has received $\$ 110$ million annually in recent years (with $\$ 10$ million in Fiscal Year 2022 allocated for NEP and the rest to NTP). Moreover, much like the RPS program, NASA and DOE have signed two MOUs, in 2016 and in 2020, to enable joint technology development of fission-based power and propulsion systems as well through an interagency agreement.

These efforts have delivered significant milestones. In 2018, NASA and DOE completed the joint Kilopower Reactor Using Stirling Technology
(KRUSTY) experiment, which aimed to assess extraterrestrial fission reactor technology for surface power. KRUSTY used highly enriched uranium fuel to successfully demonstrate heat-transfer technologies. DOE completed a reactor study in 2020 to identify reactor designs that exhibit comparable performance using low-enriched uranium, which is less expensive and more easily available. These efforts are in line with the 2020 "Memorandum on the National Strategy for Space Nuclear Power and Propulsion" Presidential Space Policy Directive. This White House document sets forth a schedule to develop a $40-\mathrm{kW}$ electric fission power generator on the lunar surface by 2027 and proceed to scale it for subsequent Mars exploration. The directive further cements DOE's role in developing reactor concepts and uranium fuel processing capabilities that will enable NASA to meet this goal.

Commercial entities are also joining the quest to deliver new nuclear propulsion capabilities. In July of 2021, NASA and DOE jointly announced three contracts, valued at $\$ 5$ million each, to BWX Technologies, Inc., General Atomics Electromagnetic Systems, and Ultra Safe Nuclear Technologies. Under these INL-managed contracts, the three companies will spend 12 months to develop conceptual nuclear propulsion reactor designs. NASA intends to advance the development of NTP using these designs.

\section{Safety and future outlook}

Naturally, production of nuclear fuel and its deployment in space, either in a radioisotope power source or a nuclear reactor, requires careful attention to safety issues and responsiveness to stakeholder concerns. Space launches are not always successful, and there is a risk of a release of nuclear material on Earth. Prior mitigation efforts have been mostly successful. The Transit IV-A satellite is now in a high-altitude graveyard orbit and will remain aloft for over 1400 years. When the malfunctioning Apollo 13 mission jettisoned its RPScontaining Lunar Lander to burn up in
Earth's orbit, the cask containing the radioisotopes, designed to withstand the heat of reentry, fell into a deep trench in the Pacific Ocean intact owing to its robust design. Owing to its protective shielding, no radioactive contents leaked out throughout the period during which the United States monitored it. Other missions were more problematic: when the former Soviet Union's Cosmos 954 satellite launch failed in 1978 , its onboard nuclear reactor scattered highly radioactive debris over large swaths of Canada. According to the Yale Journal of International Law, the cleanup cost over CAD \$14 million and spurred efforts to define international legal frameworks that govern liabilities and responsibilities for satellite accidents.

The 2019 "Launch of Spacecraft Containing Space Nuclear Systems" Presidential Memorandum outlines government agencies' policies and procedures that establishes a tiered safety process for the launch of a spacecraft with nuclear materials onboard. This Memorandum established an Interagency Nuclear Safety Review Board that includes representatives from NASA, DOE, and four other agencies. Its function is to review and identify any safety gaps prior to liftoff. However, efforts to mitigate risk start long before the spacecraft reaches the launch pad. According to Zakrajsek, during the design of any RPS-powered mission, NASA focuses on safety mechanisms of launch vehicles and space probes, while DOE ensures safety of the nuclear materials in systems that are delivered to the launch pad. Both agencies evaluate and test these configurations to understand and mitigate risks.

The mutual working relationship between NASA and DOE was recognized during the October 2021 Nuclear Science Week (NSW). The director of the National Museum of Nuclear Science \& History in New Mexico presented a Lifetime Achievement Award to the two organizations to honor their cooperative development of nuclear technology for space exploration. The 
recognition was a linchpin ceremony at the beginning of a nationwide, week-long event to promote nuclear science education. Over 250 visitors attended events that focused on the role of nuclear power in delivering clean energy and combating greenhouse gas emissions. "There are so many amazing, innovative things happening when it comes to all facets of nuclear science and the technologies propelling them into the future," said Palo Verde Nuclear Generating Station's Kory Raftery, who is the co-chair of the NSW organizing committee, in an interview with $M R S$ Bulletin. "It is extremely important that people recognize the values of these technologies, and American leadership in things like carbon-free electricity, transformative health care, and space exploration. For NSW 2021, we are truly humbled to have partners like NASA and the Department of Energy to help us showcase how nuclear sciences propel not only spacecraft and out-ofthis-world instrumentation, but also our day-to-day lives and the dreams of our future leaders."

Although efforts to develop nuclear power in space may play a role in an eventual crewed mission to Mars, planned Artemis missions to the Moon likely will rely on the same fundamental technology that had enabled the Apollo missions of the 1960s. The Space Launch System rocket that is scheduled to lift off in February of 2022 uses a conventional chemical propulsion system - as does the Blue Origin lift vehicle and all members of the
SpaceX fleet. Moreover, the Lucy mission to Jupiter Trojan asteroids relies solely on solar power, and the absence of a RTG has enabled the spacecraft to weigh less and carry additional experiments and instruments. Every trip to the exosphere must thread this needle and carefully balance power, science, and risk considerations. As NASA sets forth to reach new frontiers in space, establish permanent lunar habitats, or put first-ever boots on the ground on Mars, its missions will require evermore capable and energy-dense power sources. Nuclear power, in all its different forms, will receive significant consideration, and the corresponding NASA-DOE partnership will remain crucial.

Boris Dyatkin

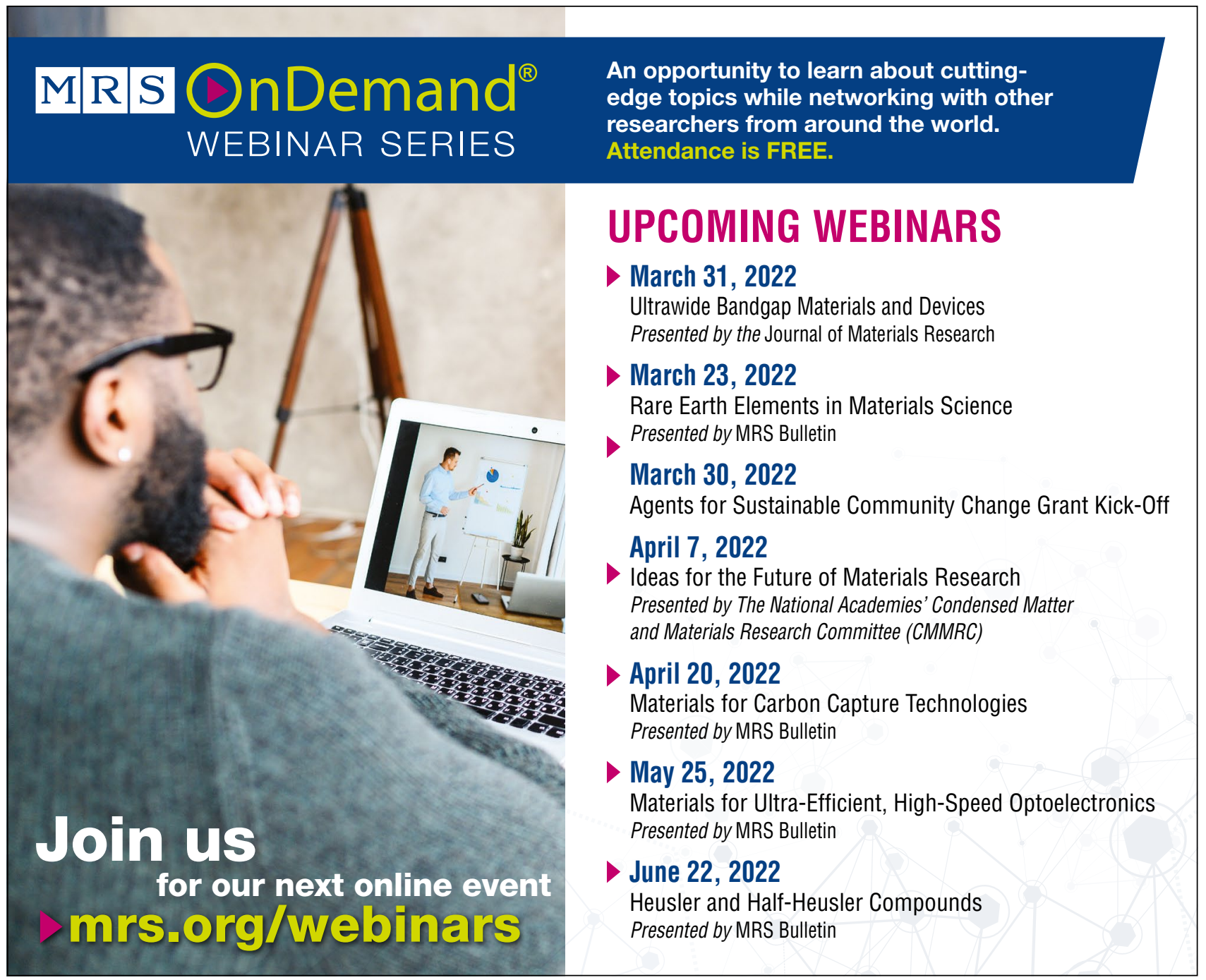

